Rev. Elev. Méd. vét. Pays trop., 1975, 28 (1) : 1-7

\title{
Valeurs comparées du B.C.G. vivant ou tué et du bacille de Koch trypsiné dans l'immunisation des zébus contre la tuberculose
}

\author{
par Y. CHENEAU $(*)$ et J. BLANCOU $\left({ }^{*}\right)$ \\ (avec la collaboration technique de D. RAMBELOMANANA)
}

\begin{abstract}
RESUME
L'inoculation de bacilles tuberculeux virulents par voie intraveineuse à 40 bovins témoins et 70 vaccinés a démontré que trois vaccins protégearent dans des proportions à peu près équivalentes :

1. Le B.C.G. vivant (100 mg, voie sous cutanée) : 17,14 à 28,5 p. 100 .

2. Le B.C.G. tué à $43^{\circ}$ (aluné à 1 p. $100,250 \mathrm{mg}$ voie sous-cutanée) : 20,91 à 25,22 p. 100 .

3. Le bacille de Koch trypsiné, aluné à 1 p. $100(50 \mathrm{mg}$, voie souscutanée) : 18,07 à 34,16 p. 100 .

Contre l'épreuve par cohabitation, ce dernier vaccin réduit l'incidence de la maladie đe 19,08 à 20,4 p. 100 , le B.C.G. ne le réduisant que de 2,1 à 12,83 p. 100 .
\end{abstract}

\section{INTRODUCTION}

Lors d'essais précédemment rapportés sur l'immunisation des zébus contre la tuberculose en élevage extensif (7) nous avions démontré sur le terrain la valeur immunogène du bacille de Koch traité par la trypsine. En même temps que ces essais préliminaires sur le terrain, des essais en Station étaient entrepris.

$\mathrm{Si}$ les premiers jugeaient de la valeur de l'immunité vis-à-vis de l'infection tuberculeuse naturelle, les seconds jugeaient de la valeur du même vaccin contre l'infection expérimentale, par voie intraveineuse.

Ce sont ces derniers que nous rapportons dans la présente note, en analysant les relations observées entre infection naturelle et infection expérimentale chez les sujets vaccinés.

Nous ne reviendrons pas ici sur les motifs qui nous ont conduits à entreprendre ces essais,

(*) Adresse actuelle: I.E.M.V.T., 10, rue Pierre Curie, 94700 Maisons-Alfort, France. déjà amplement définis précédemment $(3,7)$. J. CAROUGEAU écrivait déjà en 1911 : “ A Madagascar, la lutte contre la tuberculose doit être offensive; il faut y appliquer les méthodes procurant artificiellement l'immunisation, sans avoir besoin du concours des éleveurs, je dirai même malgré eux..." et en 1972, après K. ASPINALL, K. S. CHODNIK et F. WADDINGTON (15), R. G. MARES parlait de " those countries, where the unsophisticated nature of the farming community renders control of tuberculosis by test and slaughter politically unacceptable and impracticable » (10).

La tuberculose, maladie contagieuse tropicale, obéit donc à la règle générale que rappelle P. PERREAU : "En pays tropicaux, la vaccination reste le moyen de base, presque exclusif, pour protéger le cheptel. La prophylaxie sanitaire ne peut être entreprise que dans des circonstances particulièrement favorables... encore trop rarement réunies, et on peut le regretter $»(11)$. 


\section{MATERIEL ET METHODES}

\section{MATERIEL}

\section{Animaux et locaux d'expérience}

Tous les animaux d'expérience utilisés étaient des zébus malgaches castrés, âgés de 2 à 4 ans, pesant 275 kilogrammes en moyenne. Avant toute opération les animaux sont soumis à un examen clinique et sérologique, et à une tuberculination intradermique afin d'éliminer toute possibilité d'infection tuberculeuse préexistante (14). Cette méthode nous a donné satisfaction puisque sur 110 animaux utilisés un seul s'est révélé atteint d'une tuberculose antérieure à l'infection expérimentale.

Avant l'épreuve infectante, les animaux vaccinés sont gardés dans une station indemne de tuberculose. Le jour de l'infection expérimentale, et jusqu'à leur abattage, ils sont placés dans une étable de 32 places, isolée par une triple barrière, et munie d'un sas de décontamination (9).

\section{Types de vaccins utilisés}

Trois types de vaccins ont été utilisés, dont certains déjà décrits précédemment (7). Devant la multiplicité des " recettes " de vaccins tués, dont J. BASSET a fait une revue critique complète (2), nous avons dû nous limiter à deux de ceux qui ont été le plus longuement étudiés ou déjà utilisés en pratique courante, afin de les comparer au B.C.G.

1. B.C.G. vivant:

Nous avons employé du vaccin B.C.G. «buccal » conditionné en ampoules contenant $100 \mathrm{mg}$ de bacilles lyophilisés, titrant $10^{6}$ ger$\mathrm{mes} / \mathrm{mg}$, à leur $1.171^{\mathrm{e}}$ passage sur pomme de terre biliée (14). Il est conservé sous glace jusqu'à l'emploi.

\section{B.C.G. tué :}

Ce type de vaccin a été conseillé et utilisé à grande échelle chez l'homme au Viet-Nam (12). Nous avons utilisé le vaccin buccal précédent, conservé 50 jours à $43^{\circ}$, après avoir vérifié qu'il ne contient plus aucun élément viable.

\section{Bacille de Koch trypsiné :}

La méthode d'extraction antigénique de bacilles tuberculeux autoclavés, par la trypsine, a été longuement étudiée par A.J. CROWLE (8). Cette technique était peut-être déjà utilisée, involontairement, par J. AU-
CLAIR (1) qui, en 1930, employait avec succès un mélange de bacille tuberculeux et de pancréas de poule pour vacciner cobayes et lapins.

Nous avons adapté ce vaccin à la pratique vétérinaire en conservant les corps bacillaires digérés. Au cours d'études préliminaires, nous avons vérifié que cela ne réduisait pas la valeur immunogène de l'extrait trypsiné chez les bovins et que l'allergie induite disparaissait totalement en moins de 18 mois (9).

\section{METHODES}

\section{Vaccinations des bovins}

Les animaux reconnus non tuberculeux sont vaccinés par voie sous-cutanée, aux doses suivantes :

- B.C.G. vivant : $100 \mathrm{mg}\left({ }^{*}\right)$ en suspension extemporanée dans $5 \mathrm{ml}$ d'eau distillée neutre.

- B.C.G. tué : $250 \mathrm{mg}\left({ }^{*}\right) \mathrm{mis}$ en suspension dans $10 \mathrm{ml}$ d'eau distillée additionnée de 1 p. 100 d'alun de potassium.

- Bacille de Koch trypsiné : $50 \mathrm{mg}\left(^{*}\right)$ dans $5 \mathrm{ml}$ de l'extrait trypsiné correspondant additionné de 1 p. 100 d'alun de potassium.

Ces vaccinations ont été conduites en quatre temps successifs, permettant de comparer l'un après l'autre les trois vaccins entre eux après un délai de 75 ou de 300 jours. Pour des raisons pratiques, il était, en effet, impossible de vacciner et éprouver en même temps les 110 animaux.

Ces essais ont duré deux ans.

\section{Epreuve des bovins}

Après un temps variable selon les expériences, dix bovins vaccinés avec l'un des trois vaccins décrits sont éprouvés, en même temps que dix bovins témoins neufs de même âge et poids.

La méthode d'épreuve, retenue après plusieurs essais préliminaires (9) est l'injection intraveineuse des bacilles virulents, souche Ravenel (**).

(*) Les pesées sont effectuées après essorage des bacilles sur filtre "Seitz ".

(**) Souche aimablement fournie par Docteur A. J. Crowle du Webb-Waring Lung Institute, University of Colorado, 4200 East, 9, avenue, Denver, que nous remercions vivement à cette occasion. 


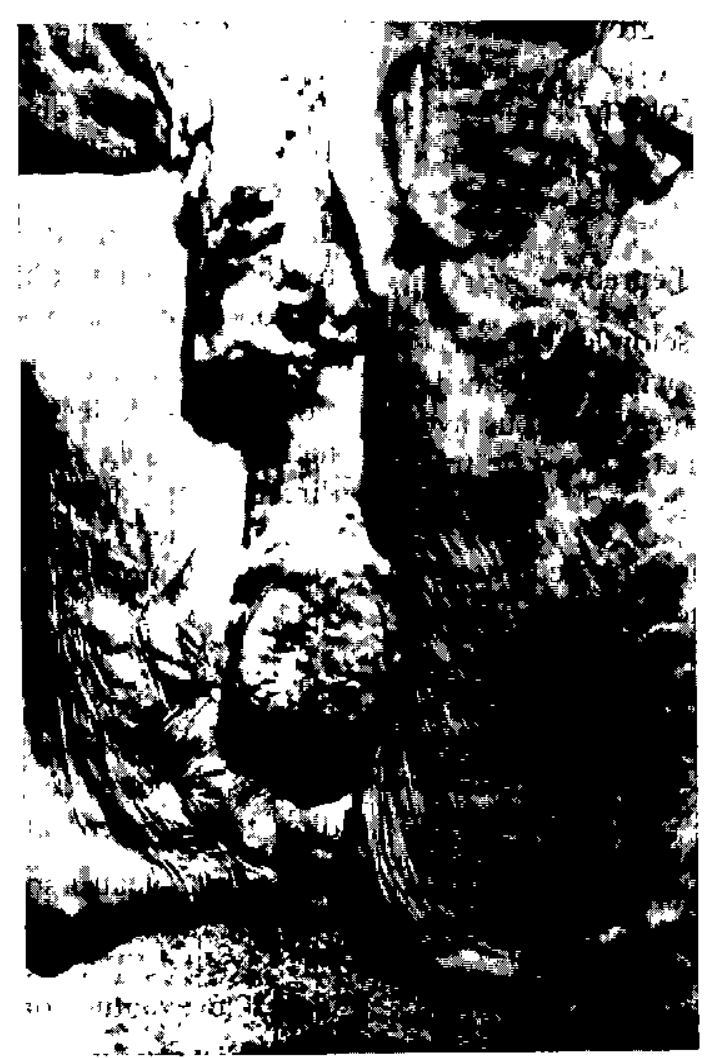

Tuberculose expérimentale du parenchyme et des ganglions pulmonaires (inoculation intraveineuse).

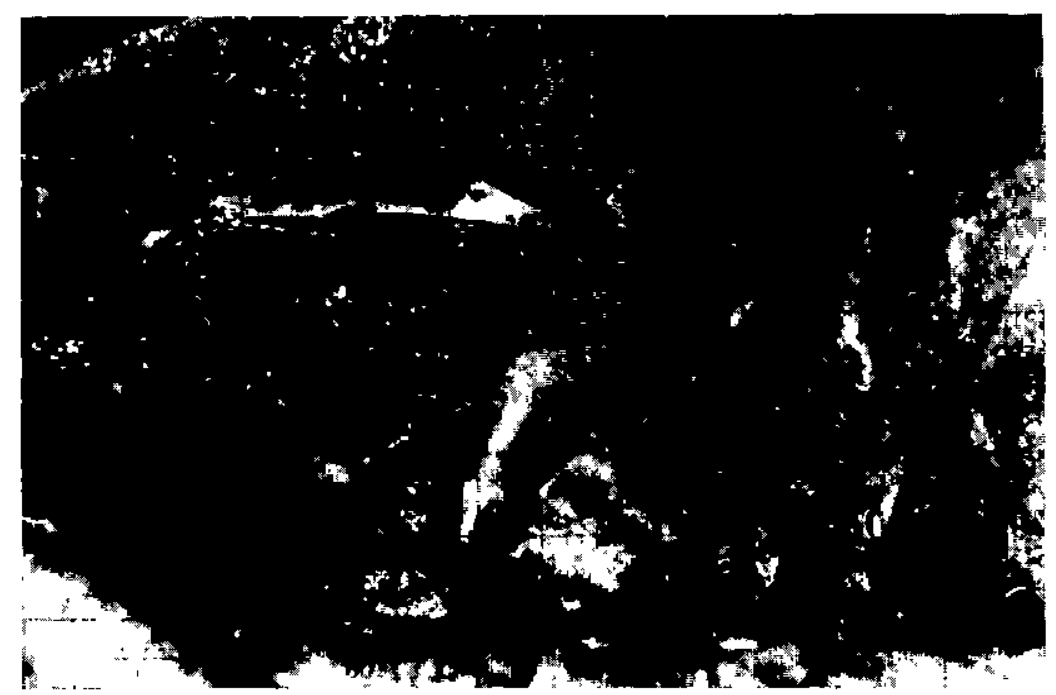

Tuberculose miliaire du parenchyme pulmonaire: coupe (inoculation intraveineuse).

Ayant constaté préalablement qu'à dose infectante égale, le développement de l'infection reste très différent selon la sensibilité individuelle des bovins, nous avons adopté une technique d'infection ménagée (9).
Chaque groupe de bovins, vaccinés et témoins, reçoit donc de 60 à $100 \mathrm{mg}$ de bacilles virulents (soit 6 à 10 millions de germes revivifiables). Ces groupes sont constitués par la "méthode des paires " qui réunit des sujets de 
même âge et poids dans le groupe vacciné et dans le groupe témoin. En sorte que, même en cas d'infection trop sévère ou trop bénigne, le degré de protection global puisse être quantifié sans difficulté.

\section{Contrôle des résultats de l'épreuve}

Après un temps variable selon les expériences (de 80 à 160 jours), les bovins sont sacrifiés. En effet, aux doses infectantes que nous avons utilisées, aucun des sujets n'était mort de tuberculose avant le $160^{\mathrm{e}}$ jour.

L'étendue de leurs lésions tuberculeuses est appréciée par examen macroscopique détaillé de la totalité du cadavre, complété par un examen histologique des poumons, du foie et du système ganglionnaire. Les lésions de tuberculose expérimentale sont le plus souvent cantonnées à ces organes (du moins dans nos délais expérimentaux) et atteignent surtout le parenchyme pulmonaire qui est parsemé de tubercules gris, puis caséeux, de 2 à $10 \mathrm{~mm}$ de diamètre. Les ganglions pulmonaires sont parfois atteints simultanément et ont un aspect "lardacé ») particulier (voir photographies).

Les résultats de ces deux examens sont quantifiés par un système de notation précédemment rapporté (7) de façon a attribuer à chacun des sujets une note globale dont le maximum varie de 9 à 11 points selon la durée de l'expérience.
Ainsi le groupe de dix témoins reçoit une note en pourcentage dite "taux de lésions" déterminée pour chaque dose infectante (6 à $10 \times 10^{6}$ bacilles). Leurs notes sont ensuite comparées aux " taux de lésions" correspondants du groupe des dix vaccinés: par différence on obtient directement le taux de protection de chaque vaccin, en pourcentage.

Cette méthode objective donne avec précision le taux de lésions évitées grâce à la vaccination. Nous la préférons à celle qui compare directement l'incidence dans les deux groupes, sans indiquer l'importance de la contamination chez les témoins.

\section{RESULTATS}

Ces résultats ne peuvent être plus facilement exposés et comparés entre eux que sous forme d'un tableau général, présenté ci-dessous.

Sur ce tableau I sont indiqués les délais après vaccination et après l'épreuve, que nous avons fait varier de 1 à 4 et de 1 à 2 afin d'observer leur rôle éventuel dans le mécanisme de protection.

\section{DISCUSSION}

\section{Valeur de la protection conférée par les vaccinations}

Si l'on admet, avec J. B. WOOLCOCK (16) que le bacille tuberculeux, parasite intracel-

TABLEAU N $^{\circ}$ I-Comparaison de la valeur des trais vaccins contre l'épreuve par voie intraveineuse.

\begin{tabular}{|c|c|c|c|c|c|}
\hline $\begin{array}{c}\text { Vaccins } \\
\text { Dose et voie } \\
\text { d'administration }\end{array}$ & $\begin{array}{l}\text { Délai } \\
\text { d'êpreuve après } \\
\text { vaccinetion (J) }\end{array}$ & $\begin{array}{c}\text { Durëe de } \\
\text { l'observation } \\
\text { après l'épreuve } \\
\text { (J.) }\end{array}$ & $\begin{array}{l}\text { Taux de } \\
\text { lë́ions chez } \\
\text { les têmoirs }\end{array}$ & $\begin{array}{l}\text { Taux de } \\
\text { lếgions chez } \\
\text { les vaccinés }\end{array}$ & $\begin{array}{l}\text { Taux de } \\
\text { protection du } \\
\text { vaccin (p.100) }\end{array}$ \\
\hline \multirow{3}{*}{$\begin{array}{l}\text { B.k. tué et } \\
\text { trypsiné, aluné } \\
\text { à } 1 \text { p. } 100 \\
50 \mathrm{mg} \text { par voie } \\
\text { soug-cut anée }\end{array}$} & \multirow[t]{2}{*}{75} & 160 & $\frac{75,71}{100}$ & $\frac{41,55}{100}$ & 34,16 \\
\hline & & 80 & $\frac{74,02}{100}$ & $\frac{55,95}{100}$ & 18,07 \\
\hline & 300 & 80 & $\frac{46,36}{100}$ & $\frac{22,72}{100}$ & 23,64 \\
\hline \multirow{2}{*}{$\begin{array}{l}\text { B.C.G. vivant } \\
100 \text { mg par voie } \\
\text { soug-cutanée }\end{array}$} & 75 & 160 & $\frac{75,71}{100}$ & $\frac{47,14}{100}$ & 28,57 \\
\hline & 300 & 80 & $\frac{54,28}{100}$ & $\frac{37,14}{100}$ & 17,14 \\
\hline \multirow{2}{*}{$\begin{array}{l}\text { B.C.G. tuê à } \\
43^{\circ} \text {, aluné } \\
\text { à 1 p. } 100 \\
250 \text { mg par voie } \\
\text { sous-cutanée }\end{array}$} & 75 & 80 & $\frac{74,02}{100}$ & $\frac{48,80}{100}$ & 25,22 \\
\hline & 300 & 80 & $\frac{46,36}{100}$ & $\frac{25,45}{100}$ & 20,91 \\
\hline
\end{tabular}


lulaire facultatif, ne peut être neutralisé que par une immunité à médiation cellulaire, seul un vaccin vivant (B.C.G.) ou un vaccin à antigènes " démasqués " par une digestion cellulaire ou trypsinique pourrait être efficace. Mais, même dans ces conditions, on ne doit pas prévoir que la vaccination antituberculeuse dépasse le but que lui fixe A. J. CROWLE : " to blunt the almost inevitable primary infection and converts the potentially dangerous infection into a natural reimmunization " (8).

L'analyse statistique de ces résultats montre que les taux de protection conférés par les trois vaccins sont tous significatifs à $t_{0,01}$ ou $t_{0,05}$.

Par contre, il n'y a pas de différence significative entre les différents taux de protection.

La valeur statistique de l'écart entre les taux de lésions des sujets vaccinés et témoins étant nettement établie, il apparaît que le taux de protection conféré par les trois vaccins est peu élevé (variant de 17,14 à 34,16 p. 100). Ceci n'est pas pour surprendre en matière de tuberculose, dans ce type d'épreuve.

En effet, hormis l'expérience princeps de CALMETTE (5) où le taux de protection conféré était de 100 p. 100 (la souche B.C.G. n'étant qu'à son $33^{\mathrm{e}}$ passage), les taux de protection observés ultérieurement ont toujours été de cet ordre. Ainsi que l'indique J. BASSET (2): “Si l'épreuve est mortelle pour les témoins, tous les vaccinés au B.C.G. deviennent tuberculeux et la moitié d'entre eux meurent comme les témoins."

Plus surprenant est le bon résultat obtenu avec les vaccins tués.
Il est probablement dû au traitement par la trypsine dans un cas et la forte dose de B.C.G. tué dans l'autre. L'addition d'alun de potassium favorise aussi sûrement l'installation de l'immunité, et compense l'absence de multiplication in vivo de la souche vaccinale.

L'inconvénient des réactions locales provoquées par cet adjuvant (induration et parfois abcès froids) est compensé par l'avantage incontestable du caractère thermostable du vaccin.

Remarque: Il est intéressant de noter que :

- Le taux de protection est toujours plus net lorsque la durée d'observation des animaux est plus longue, l'évolution des lésions chez les témoins s'accentuant au cours du temps;

- Le taux de protection varie très peu dans les 300 jours qui suivent la vaccination malgré la disparition relativement rapide de l'allergie (7).

\section{Comparaison entre les taux de protection conférés lors de l'épreuve par voie intraveineuse et de l'épreuve par cohabitation}

Cette comparaison n'a pu, malheureusement, être réalisée pour les trois vaccins. Mais elle l'a été de façon complète pour le vaccin trypsiné et Ie B.C.G. vivant.

Pour permettre de juger de la corrélation entre ces deux taux de protection contre l'épreuve artificielle et l'épreuve naturelle, nous en reproduisons ci-dessous un tableau comparatif :

TABLEAU $N^{\bullet} I I-C o m p a r a i s o n$ entre les taux de protection.

\begin{tabular}{|c|c|c|}
\hline Vaccins & Epreuve par cohabitation & Epreuve par voie intraveineuse \\
\hline \multirow{2}{*}{$\begin{array}{l}\text { Vaccin trypsiné } \\
\text { aluné à lp.100 } \\
50 \text { mg par voie } \\
\text { sous-cutanée. }\end{array}$} & $\begin{array}{l}\text { Première expérience sur } \\
111 \text { bovins (7) : } \\
20,4 \quad \text { P.100 }\end{array}$ & $\begin{array}{c}75 \text { jours après vaccination : } \\
18,07 \text { à } 34,6 \mathrm{p} .100\end{array}$ \\
\hline & $\begin{array}{c}\text { Seconde expërience sur } \\
138 \text { bovins: } \\
19,08 \text { p. } 100\end{array}$ & $\begin{array}{c}300 \text { jours après vaccination : } \\
23,64 \text { p. } 100\end{array}$ \\
\hline \multirow{2}{*}{$\begin{array}{l}\text { B.C.G. vivant } \\
100 \mathrm{mg} \text { par voie } \\
\text { sous-cutanée. }\end{array}$} & $\begin{array}{c}\text { Premiẽre expérience sur } \\
116 \text { bovins (7): } \\
2,1 \text { p.100 }\end{array}$ & $\begin{array}{l}75 \text { jours après la vaccination : } \\
28,57 \text { p. } 100\end{array}$ \\
\hline & $\begin{array}{l}\text { Seconde expërience sur } \\
97 \text { bovins : } \\
12,63 \text { p.100 }\end{array}$ & $\begin{array}{c}300 \text { jours après vaccination : } \\
17,14 \mathrm{p}, 100\end{array}$ \\
\hline
\end{tabular}




\section{Remarque :}

En ce qui concerne les taux de protection conférés contre l'épreuve "par cohabitation" nous citons d'une part les chiffres rapportés au cours de nos essais préliminaires (7), d'autre part ceux obtenus au cours d'une expérience exactement similaire faite l'année suivante (9).

On constate clairement que les taux de protection, quelle que soit l'épreuve choisie, sont très proches l'un de l'autre, en ce qui concerne le vaccin trypsiné. En ce qui concerne le B.C.G., sa valeur sur le terrain est plus décevante mais toutefois moins que lors de notre expérimentation préliminaire (7).

Si l'on admet que la protection conférée contre l'infection naturelle est plus fiable que celle conférée contre l'inoculation intraveineuse, il n'en reste pas moins que cette dernière, plus facile à mettre en œuvre, donne des résultats plus rapides et des indications valables sur l'efficacité relative de vaccins comparés entre eux.

\section{CONCLUSION}

La vaccination antituberculeuse offre une protection réduite contre cette affection chronique. Cette protection semble acquise aussi bien par l'injection de la souche atténuée B.C.G. vivante $(100 \mathrm{mg})$ que par celle de la même souche tuée par chauffage à $43^{\circ}$ mais à dose supérieure $(250 \mathrm{mg}$ ) ou de bacilles virulents trypsinés $(50 \mathrm{mg})$. Dans ces deux derniers cas, il convient d'ajouter un adjuvant de l'immunité aux corps bacillaires injectés : l'alun de potassium à 1 p. 100.

Le taux de protection conféré semble à peu près identique contre l'épreuve artificielle (injection intraveineuse) et contre l'épreuve naturelle (par cohabitation). Dans ce dernier cas, le vaccin trypsiné peut réduire la morbidité de 19 à 20 p. 100.

Cette observation, jointe à l'intérêt majeur que présente sa thermostabilité, en ferait le vaccin de choix d'une prophylaxie médicale en élevage extensif. Ainsi semble en bonne voie de réalisation le vœu que formait G. RAMON en 1951 (13) :

« Les problèmes que posent ainsi le B.C.G. et sa propre amélioration étant difficilement solubles, il faut, à notre avis, chercher la solution dans une autre formule de vaccins, par exemple des vaccins constitués soit par des germes totalement inactivés au moyen d'agents physiques ou chimiques, dans leur vitalité, dans leur virulence, dans leur pouvoir pathogène et dont on puisse accroître la valeur antigénique, les doses et par conséquent la valeur immunisante sans nuire en aucune façon à leur innocuité, soit encore par des antigènes extraits de bacilles, ces vaccins méritant le nom de " vaccins chimiques" (Pasteur) privés de vie, incapables de se reproduire et de varier dans leurs propriétés ".

\section{SUMMARY}

\section{Immunizing value of B.C.G. (alive or heat-killed) and trypsin-extracted tubercle bacilli in cattle tuberculosis}

110 cattle (40 controls and 70 vaccinated) were challenged intravenously by virulent tubercle bacilli.

Three vaccins gave the same protection:

1. B.C.G. alive (100 mg, s.c. route): 17,4 to 28,5 p. 100.

2. B.C.G. heat killed plus Potash alum (250 mg): 20,91 to 25,22 p. 100 .

3. B.K. trypsin extracted plus Potash alum (50 mg): 18,07 to 34,16 p. 100.

Trypsin extracted vaccin gave 19,08 to 20,4 p. 100 protection in field trials and B.C.G. (alive) 2,1 to 12,83 p. 100 . 


\section{RESUMEN}

\section{Comparación de los valores del B.C.G. viviente o matado y del bacilo de Koch tripsinado utilizados para la inmunización de cebues contra la tuberculosis}

La inoculación de bacilos tuberculosos virulentos por via intravenosa en 40 bovinos testigos y 70 vacunados demostró que tres vacunas tenian una acción inmunizante poco más o menos equivalènte :

- El B.C.G. viviente (100 mg, por via subcutánea): 17,14 a 28,5 p. 100 ;

- E1 B.C.G. matado a $43^{\circ}$ (con addición de 1 p. 100 de alumbre, $250 \mathrm{mg}$ por vía subcutánea): 20,19 a 25,22 p. 100 ;

- El bacilo de Koch tripsinado, con addición de 1 p. 100 de alumbre (50 mg por via subcutánea): 18,07 a $34,16 \mathrm{p} .100$.

Contra la prueba por cohabitación, la última vacuna reduce la incidencia de la enfermedad de 19,08 a 20,4 p. 100 , el B.C.G. reduciendolo solamente de 2,1 a 12,83 p. 100 .

\section{BIBLIOGRAPHIE}

1. AUCLAIR (J.). Vaccination préventive et curative du cobaye et du lapin contre la tuberculose humaine. Paris, Masson, 1930.

2. BASSET (J.). Immunologie et prophylaxie de la tuberculose chez l'homme et chez le bouf. Paris, Vigot Frères, 1953.

3. BLANCOU (J.), ROHRBACH (C.), PERDRIX (A.), CHOQUEL (P.) et ROSNER (G.). La tuberculose bovine à Madagascar. Rev. Elev. Méd. vêt. Pays trop., 1971, 24 (4) : 505-517.

4. BLANCOU (J.). Comparaison de techniques pratiques de diagnostic de la tuberculose bovine. Rev. Elev. Méd. vét. Pays trop., 1972, 25 (1): 29-35.

5. CALMETTE (A.). La vaccination préventive contre la tuberculose par le B.C.G. Paris, Masson, 1927.

6. CAROUGEAU (J.). Tuberculose des animaux domestiques à Madagascar. Bull. Soc. Sci. méd. Madagascar, 1911 (4): 75-98.

7. CHENEAU (Y.), BLANCOU (J.) et SERRES $\left(H_{1}\right)$. Essais préliminaires d'immunisation des zébus d'élevage extensif contre la tuberculose. Rev. Elev. Méd. vét. Pays trop., 1973, 26 (2): 141-145.

8. CROWLE (A, J.). Trypsin-extracted immunizing antigen of the tubercle Bacillus : A practical vaccine? Advances in Tuberculoses Research, 1972, 18 : 31-102.
9. Détermination des moyens à mettre en cuvre pour l'éradication de la tuberculose bovine à Madagascar. 1974. Rapport du Marché $2 / 71$ FACl FNDE-DIR/EL. (Archives I.E.M.V.T.)

10. MARES (R. G.). Control of bovine tuberculosis in Malawi by the use of B.C.G. vaccination. Vet. Rec., 1972, 90 (15) : 429.

11. PERREAU (P.). Maladies tropicales du bétail. Paris, P.U.F., 1973. (Coll. Techniques vivantes).

12. PHAM NGOC THACH, NGUYEN THI HEI DANE DUC TRACH, TANG HUU THANH et NGUYEN DINH HUONG. L'immunisation contre la tuberculose par le B.C.G. tué. Rev. I $\mathrm{mmu}$ nol. Thér. antimicrob., 1963, 32 (1-2): 77-90.

13. RAMON (G.). La lutte préventive contre la tuberculose.

I. Immunité et allergie en matière de tuberculose. II. Cas particulier de la prophylaxie de la tuberculose bovine: vaccination et mesures sanitaires. Bull. off. int. Epiz., 1951, 35 (3-4) : 113-176.

14. Rapports annuels du laboratoire de l'Elevage. Tananarive, Madagascar (Archives I.E.M.V.T.).

15. WADDINGTON (F. G.) et ELLWOOD (D. C.). An experiment to challenge the resistance to tuberculosis in B.C.G. vaccinated cattle in Malawi. Brit. vet. J., 1972,128 (11): 541-552.

16. WOOLCOCK (J. B.). Resistance to microbial infection-vaccines in theory and practice. Aust. vet. J., 1973, 49 (6) : 307-317. 